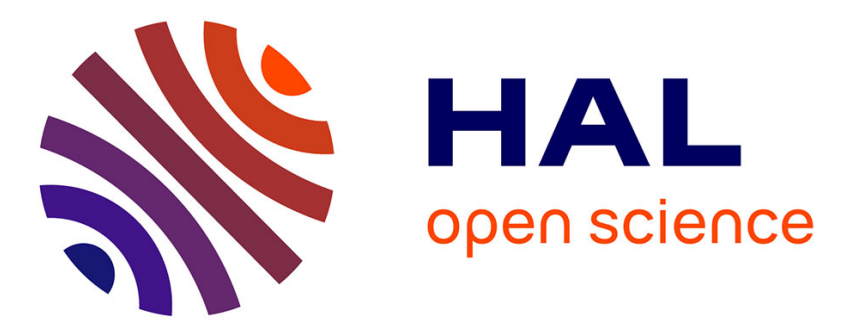

\title{
Reinterpreting and Extending Anatol Vieru's Periodic Sequences Through the Cellular Automata Formalisms
}

Paul Lanthier, Corentin Guichaoua, Moreno Andreatta

\section{To cite this version:}

Paul Lanthier, Corentin Guichaoua, Moreno Andreatta. Reinterpreting and Extending Anatol Vieru's Periodic Sequences Through the Cellular Automata Formalisms. Mathematics and Computation in Music, 7th International Conference, MCM 2019, Madrid, Spain, June 18-21, 2019, pp.261-272, 2019, 10.1007/978-3-030-21392-3_21. hal-02372515

\section{HAL Id: hal-02372515 https://hal.science/hal-02372515}

Submitted on 20 Nov 2019

HAL is a multi-disciplinary open access archive for the deposit and dissemination of scientific research documents, whether they are published or not. The documents may come from teaching and research institutions in France or abroad, or from public or private research centers.
L'archive ouverte pluridisciplinaire HAL, est destinée au dépôt et à la diffusion de documents scientifiques de niveau recherche, publiés ou non, émanant des établissements d'enseignement et de recherche français ou étrangers, des laboratoires publics ou privés. 


\title{
Reinterpreting and extending Anatol Vieru's Periodic Sequences through the Cellular Automata formalisms
}

\author{
Paul Lanthier ${ }^{1}$, Corentin Guichaoua ${ }^{2}$, and Moreno Andreatta ${ }^{3,2}$ \\ 1 Université de Rouen, LMRS (UMR 6085) \\ paul.lanthier@etu.univ-rouen.fr \\ 2 Université Strasbourg, USIAS/IRMA (UMR 7501) \\ \{guichaoua, andreatta\}@math.unistra.fr \\ 3 IRCAM/CNRS/Sorbonne Université, France \\ moreno.andreatta@ircam.fr
}

\begin{abstract}
In this paper we focus on Anatol Vieru's periodic sequences that we approach with the formalism of the theory of cellular automata. After extending previous results about the action (in the image direction) of one particular cellular automaton on periodic sequences we show the existence of a second one which is its complementary (or dual). The main idea of the paper is that the study of preimages of one of those two automata is the study of the images of the other one and vice versa. By using the duality, we have been able to show explicitly the evolution of the period and the form of the preimages for both automata. In order to illustrate the theoretical constructions, a musical composition is presented using the two automata both in the image and preimage directions.
\end{abstract}

\section{Introduction}

The theory of periodic sequences has been introduced by the Romanian composer Anatol Vieru (1926-1998) in the 1980's [5] as an original way to approach "modalism" as a third World [4] between tonality and atonality. This approach has been firstly formalized by mathematician Dan T. Vuza in his systematic mathematical study of the modal theory of Anatol Vieru [6]. Vieru's constructions have been successively investigated and developed at the end of the Twentieth Century, thanks to increasing collaborations between mathematicians, computer sciences and computational musicologists 23 .

Our study tries to follow this path by taking into account the most recent results on the algebraic formalization of Vieru's Periodic sequences [1] and confronting the traditional group- and module-theoretical approach with the computational framework provided by cellular automata. This new formalism also applies to the algebraic formalization of the equal-tempered system assuming that a given cyclic group of order $n$ may represent, at the same time, the collection of pitches (or pitch classes) and intervals (or interval classes). The finite 
difference calculus applied to periodic sequences taking values in a cyclic group is not the only natural construction enabling the composer to explore the intimate relation between notes and intervals. There is a similar operator which is widely used in cellular automata theory and which considers additions between consecutive elements of a periodic sequence taking value in a given cyclic group.

One of the main ideas of this paper is to explore the existing duality between those two automata, using some ideas coming both from the classical algebraic approach and from the new framework based on cellular automata. The main problem, which has been partially solved, concerns the complete characterization of reducible and reproducible components of a periodic sequence in the case of the additive operator as well as study of the inverse temporal process of both the difference and additive finite calculus. This question has rarely been addressed in the previous literature despite its natural compositional application. The study of the preceding layers of a given periodic sequence musically corresponds to the search of a melodic patterns which are able to generate a given musical theme after a given number of iterations of the finite additive or difference calculus operator. These layers depend on the choice of the initial element of every preceding sequence and this can be done in a deterministic or a random way.

In order to illustrate the music-theoretical and computational properties of this inverse construction, a microtonal fugue has been composed. The subject, or main theme of the fugue, and its imitations structurally interact thanks to the action of the main automata (associated respectively with the finite difference and finite addition calculus) in the past and the future history of the main theme. This shows the reappearance of some elements of the main theme and their imitations at different preceding layers and permits to make use of them in the development of the canonic process.

\section{Background}

\subsection{Cellular automata}

Cellular automata are specific transformations acting on a finite or infinite grid of cells. The fondamental principle is that each cell interacts with its close neighbourhood according to the rule of the cellular automaton. Let $\mathcal{A}$ be a finite set called alphabet and $E$ the grid, a countable set. We call "full-shift" $\mathcal{X}=\mathcal{A}^{E}$ the set of all the possible configurations. We will choose $E=\mathbb{Z}$.

Let $\mathcal{N} \subset \mathbb{Z}$ finite be our neighbourhood, it describes the size of the interaction between cells over the action of the automaton.

A basic transformation is called the "shift", $\sigma: \mathcal{X} \rightarrow \mathcal{X}$ defined locally by:

$$
(\sigma x)(i)=x(i+1), \text { for } x \in \mathcal{X} .
$$

A determinist cellular automaton is a continuous transformation $F: \mathcal{X} \rightarrow \mathcal{X}$ defined by a neighbourhood $\mathcal{N}=\left\{k_{0}, \ldots, k_{n}\right\}$, a finite subset of $\mathbb{Z}$ and a local transformation $f: \mathcal{A}^{\mathcal{N}} \rightarrow \mathcal{A}$ which commutes with the shift $\sigma: \sigma \circ F=F \circ \sigma$ and defined by

$$
\forall i \in \mathbb{Z}:(F x)(i)=f\left(x\left(i+k_{0}\right), \ldots, x\left(i+k_{n}\right)\right):=f(x(i+\mathcal{N})) .
$$


We note DCA the set of deterministic cellular automata.

\section{Definition 1 (Spatial dependence cone).}

Let $Y:=\left(X_{n}(i)_{i \in \mathbb{Z}}\right)_{n \in \mathbb{Z}} \subset \mathcal{X}$ a given family of configurations. We define the spatial dependence cone of $Y$ for a given neighbourhood $\mathcal{N}:=\left\{k_{0}, \ldots, k_{n}\right\}$, noted $\mathcal{S}_{\mathcal{N}}^{Y}$, localised by a spatial point $(i, n) \in \mathbb{Z}^{2}$ and bounded by a length $\ell \in \mathbb{N}$ as follows:

$$
\mathcal{S}_{\mathcal{N}}^{Y}(i, n, \ell):=\left\{(m, j) / m \in \llbracket n, n+\ell \rrbracket:(m-n) k_{0}+i \leq j \leq(m-n) k_{n}+i\right\} .
$$

Property 1 (Trivial property). Let $F: \mathcal{X} \rightarrow \mathcal{X} \in$ DCA with $f$ its local rule and $\mathcal{N}$ its neighbourhood. Let $Y:=\left(X_{n}(i)_{i \in \mathbb{Z}}\right)_{n \in \mathbb{Z}} \subset \mathcal{X}$ with $\mathcal{S}_{\mathcal{N}}^{Y}$ its dependence cone. If $\forall(j, m) \in \mathcal{S}_{\mathcal{N}}^{Y}(i, n, \ell): X_{m}(j)=f\left(X_{m-1}(j+\ell)_{\ell \in \mathcal{N}}\right)$, then $X_{n}(i)=F^{\ell} X_{n-\ell}(i)$.

We shall now define an automata class that we will study hereafter.

Definition 2 ( $\sigma$-polynomial deterministic cellular automata). We note $\mathbb{Z}\left[\sigma^{ \pm 1}\right]$ the set of $\sigma$-polynomial automata, i.e., of the form $F=\sum_{i \in \mathbb{Z}} a_{i} \sigma^{i}$, where a finite number of $a_{i} \in \mathbb{Z}$ are non-zero and $\sigma^{i}$ is the ith self-composition of $\sigma$.

The automata belonging to $\mathbb{Z}\left[\sigma^{ \pm 1}\right]$ are linear : Let $F_{1}, F_{2} \in \mathbb{Z}\left[\sigma^{ \pm 1}\right]$ from a set $\mathcal{X}$ in itself, then $\forall x \in \mathcal{X}:\left(F_{1}+F_{2}\right)(x)=F_{1}(x)+F_{2}(x)$ and as $\mathbb{Z}\left[\sigma^{ \pm 1}\right] \subset$ DCA we have that $\forall F_{1}, F_{2} \in \mathbb{Z}\left[\sigma^{ \pm 1}\right]: F_{1} \circ F_{2}=F_{2} \circ F_{1}$ as $F \circ \sigma=\sigma \circ F$.

Two of these automata will be of particular focus to our work:

$$
\left\{\begin{array} { r l } 
{ \Delta = \sigma - I d } \\
{ f _ { \Delta } : \mathcal { A } \times \mathcal { A } \rightarrow \mathcal { A } } \\
{ ( a , b ) \mapsto b - a }
\end{array} \quad \text { and } \quad \left\{\begin{array}{r}
\tau=\sigma+I d \\
f_{\tau}: \mathcal{A} \times \mathcal{A} \rightarrow \mathcal{A} \\
(a, b) \mapsto b+a
\end{array}\right.\right.
$$

Both are of neighbourhood $\mathcal{N}=\{0,1\}$, the first one beeing the discrete derivative (the one studied by A.Vieru) and the other one the well-known Ledrappier's one.

However, the first few properties we show apply to all such automata.

Property 2 (Isomorphism with the ring of Laurent polynomials).

$\left(\mathbb{Z}\left[X^{ \pm 1}\right],+, \times\right) \sim\left(\mathbb{Z}\left[\sigma^{ \pm 1}\right],+, \circ\right)$, thus $\left(\mathbb{Z}\left[\sigma^{ \pm 1}\right],+, \circ\right)$ is a commutative ring.

Proof. We exhibit the isomorphism :

$$
\begin{aligned}
\varphi: \mathbb{Z}\left[X^{ \pm 1}\right] & \rightarrow \mathbb{Z}\left[\sigma^{ \pm 1}\right] \\
\left(\sum_{i} a_{i} X^{i}\right)+\left(\sum_{i} b_{i} X^{i}\right) & \mapsto\left(\sum_{i} a_{i} \sigma^{i}\right)+\left(\sum_{i} b_{i} \sigma^{i}\right) \\
\left(\sum_{i} a_{i} X^{i}\right) \times\left(\sum_{i} b_{i} X^{i}\right) & \mapsto\left(\sum_{i} a_{i} \sigma^{i}\right) \circ\left(\sum_{i} b_{i} \sigma^{j}\right)
\end{aligned}
$$

We can see that this isomorphism is compatible with the two laws of the ring of Laurent polynomials.

We now present on which space of state $\mathcal{X}$ we want to work on. 


\section{$2.2 \quad$ Periodic sequences}

This section introduces the notation we will use throughout the article.

For the purpose of this article, a sequence will be a function $X: \mathbb{Z} \rightarrow \mathbb{Z}_{N}$, and $\left(\mathbb{Z}_{N}^{\mathbb{Z}},+, \times\right)$ is the ring of $\mathbb{Z}_{N}$-valued sequences.

Definition 3 (Periodic sequence). A sequence $X$ is periodic if there exists a positive integer $\tau$ such that $\forall i, X(i)=X(i+\tau)$. The set of periodic sequences is noted $\mathcal{C}_{N}$.

Definition 4 (Cyclicity and period). Let $X \in \mathcal{C}_{N}^{\pi}$. Any positive integer $\pi$ such that $\forall i, X(i)=X(i+\pi)$ is called a cyclicity of $X$. The smallest such integer $\tau$ is its period. We will say that $X$ is $\pi$-cyclic and $\tau$-periodic respectively The set of $\pi$-cyclic sequences is noted $\mathcal{C}_{N}^{\pi}$.

Definition 5 (Reducibility). Let $F \in \mathbb{Z}\left[\sigma^{ \pm 1}\right]$. A sequence $X \in \mathcal{C}_{N}^{\pi}$ is said to be reducible over $F$ if and only if there is an integer $\delta$ such that $F^{\delta} X=0^{\mathbb{Z}}$. The set of such sequences is $\operatorname{Red}_{F}\left(\mathcal{C}_{N}^{\pi}\right)=\bigcup_{i \in \mathbb{N}} \operatorname{ker}\left(F^{i}\right)$.

Definition 6 (Reproducibility). Let $F \in \mathbb{Z}\left[\sigma^{ \pm 1}\right]$. A sequence $X \in \mathcal{C}_{N}^{\pi}$ is said to be reproducible over $F$ if and only if there is a positive integer $\delta$ such that $F^{\delta} X=X$. The set of such sequences is $\operatorname{Rep}_{F}\left(\mathcal{C}_{N}^{\pi}\right)=\bigcup_{i \in \mathbb{N}} \operatorname{ker}\left(F^{i}-i d\right)$.

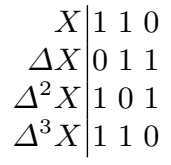

(a) A reproducible sequence on $\mathbb{Z}_{2}$
$X \mid 1351$

$\Delta X \mid \begin{array}{lllll}\Delta & 2 & 2 & 2\end{array}$

$\Delta^{2} X \mid \begin{array}{llll}0 & 0 & 0 & 0\end{array}$

(b) A reducible sequence on $\mathbb{Z}_{6}$

Theorem 1. Let $F \in \mathbb{Z}\left[\sigma^{ \pm 1}\right]$ and $\pi \in \mathbb{N}^{*}$. Any periodic sequence $X \in \mathcal{C}_{N}^{\pi}$ can be decomposed uniquely as $X=X_{\text {red }}+X_{\text {rep }}$, where $X_{\text {red }} \in \operatorname{Red}_{F}\left(\mathcal{C}_{N}^{\pi}\right)$ and $X_{\text {rep }} \in \operatorname{Rep}_{F}\left(\mathcal{C}_{N}^{\pi}\right)$. In other terms, $\mathcal{C}_{N}^{\pi}=\operatorname{Rep}_{F}\left(\mathcal{C}_{N}^{\pi}\right) \oplus \operatorname{Red}_{F}\left(\mathcal{C}_{N}^{\pi}\right)$.

Proof. The proof of [2, th3] generalises directly, replacing occurrences of $\Delta$ by any polynomial automaton $F$.

\section{Summary of previous results and extensions on the automata $\tau$ and $\Delta$}

We will henceforth focus on the automata $\tau$ and $\Delta$. To maintain brevity for shared properties, we will assume $F$ to stand for either.

\footnotetext{
${ }^{4}$ This is slightly contrasting with conventional terminology in which saying a sequence is $\tau$-periodic does not imply minimality.
} 


\subsection{Existing results on images of $\Delta$}

Now that we have defined the notions of reproducibility and reducibility, a topic of interest is their characterisation. Several results exist on $\Delta$, but $\tau$ has yet to be studied extensively.

Theorem 2 ([2, th. 7]). Let $X$ be a $\mathbb{Z}_{p^{k}}$ valued sequence, with $p$ prime. $X$ is reducible if and only if it is $p^{m}$-periodic.

While in appearance this result only applies to some cases, because $\mathbb{Z}_{N}$ (and by extension $\mathcal{C}_{N}^{\pi}$ ) can be decomposed as a direct sum of its $p$-maximal subgroups, any sequence can be decomposed as one or more such case. [1] refined this result with the addition of bounds on the number of steps needed to reduce the sequence, which we do not detail here.

We note $\eta_{a, b}$ such that $\forall X \in \mathbb{Z}_{N}^{\mathbb{Z}}, \eta_{a, b} X(i)=X(a i+b)$.

Theorem 3. Let $X \in \mathcal{C}_{N}^{\pi}$. If $X$ is reducible over $\Delta$, then $\eta_{a, b} X$ is also reducible over $\Delta$. Furthermore, if $a$ and $\pi$ are coprime, then the converse holds.

Proof. The first part is a corollary of the previous theorem. The second part follows from the fact that if $a$ and $\pi$ are coprime, then $\eta_{a, b} X$ is a permutation of $X$, so $X=\eta_{a, b}^{n} \eta_{a, b} X$ for some $n$.

Theorem 4. Let $X \in \mathcal{C}_{p^{k}}^{\pi}$ with $p$ prime. $X$ is reproducible if and only if $\forall b \in$ $\llbracket 1, \pi \rrbracket, \eta_{p^{r}, b} X$ is reproducible, with $r$ the maximum integer such that $p^{r}$ divides $\pi$.

Proof. This is essentially a reformulation of corollary 15 of [2], with the added step of re-applying the parent theorem to the condition in order to express it in terms of reproducibility of its subsequences.

\subsection{New results on preimages}

We now consider the preimages of a given sequence through $\Delta$ and $\tau$. We can rewrite the local relation defined by $\Delta$ as $X(i+1)=X(i)+\Delta X(i)$ and that defined by $\tau$ as $X(i+1)=X(i)-\tau X(i)$, thus determining a single value of an antecedent of a given sequence determines all of its values.

We will note $\Delta_{a}^{-1}$ (respectively $\tau_{a}^{-1}$ ) the function that maps a sequence to its preimage through $\Delta$ (respectively $\tau$ ) with value $a$ at index 0 . By extension, we will note $\Delta_{A}^{-n}$ with $A=\left(a_{1}, \ldots, a_{n}\right)$ the function that maps $X$ to $X^{\prime}$ such that $\Delta^{n} X^{\prime}=X$ and $\forall j \in \llbracket 1, n \rrbracket, \Delta^{n-j} X^{\prime}(0)=a_{j}$. We also note for $X \in \mathbb{Z}_{N}^{\mathbb{Z}}$ :

$$
F_{X}^{n}= \begin{cases}F^{n} & \text { if } n \geq 0 \\ F_{X(n)}^{-1} \circ F_{X}^{n+1} & \text { if } n<0\end{cases}
$$

For $n$ increasingly negative the process can be either deterministic or random depending on how the initial values of the preimages are chosen. 


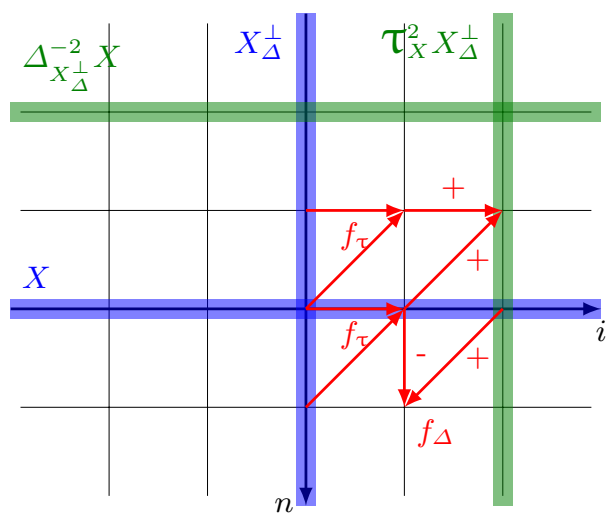

Fig. 2: Illustration of the notations. The short arrows show the local rules of automata, and wide coloured lines are sequences.

Theorem 5 (Duality of $\Delta$ and $\tau$ ). Let $X_{F}^{\perp} \in \mathbb{Z}_{N}^{\mathbb{Z}}$ be a sequence such that $X_{F}^{\perp}(i)=F^{i} X(0)$ for all positive $i$. We call it a dual of $X$. Then $\forall i, n \in \mathbb{Z}$, we have $\Delta_{X_{\Delta}^{\perp}}^{n} X(i)=\tau_{X}^{i} X_{\Delta}^{\perp}(n)$ and $\tau_{X_{\boldsymbol{\tau}}^{\perp}}^{n} X(i)=\Delta_{X}^{i} X_{\boldsymbol{\tau}}^{\perp}(n)$.

Proof. We write the proof for $F=\Delta$ but it also stands for $F=\tau$ as well. Let $Y:=\left(\Delta_{X_{\Delta}^{\perp}}^{n} X(i)_{i \in \mathbb{Z}}\right)_{n \in \mathbb{Z}}$ and $Y^{\perp}:=\left(\Delta_{X_{\Delta}^{\perp}}^{n} X(i)_{n \in \mathbb{Z}}\right)_{i \in \mathbb{Z}}$. Let $\mathcal{S}_{\mathcal{N}}^{Y}$ be the dependence cone of $Y$ and let $\mathcal{S}_{\mathcal{N}}^{{ }^{\perp}}$ be the dependence cone of $Y^{\perp}$. We first remark that $\forall i, n \in \mathbb{Z}, \ell \in \mathbb{N}: \mathcal{S}_{\mathcal{N}}^{Y}(i, n, \ell)=\mathcal{S}_{\mathcal{N}}^{{ }^{\perp}}(n, i, \ell) . \forall(j, m) \in \mathcal{S}_{\mathcal{N}}^{X}(i, n, l)$ we have :

$$
\begin{aligned}
\Delta_{X_{\Delta}^{\perp}}^{m} X(j) & =f_{\Delta}\left(\Delta_{X_{\Delta}^{\perp}}^{m-1} X(j), \Delta_{X_{\Delta}^{\perp}}^{m-1} X(j+1)\right) \\
& =\Delta_{X_{\Delta}^{\perp}}^{m-1} X(j+1)-\Delta_{X_{\Delta}^{\perp}}^{m-1} X(j) \\
\text { So } \Delta_{X_{\Delta}^{\perp}}^{m-1} X(j+1) & =f_{\tau}\left(\Delta_{X_{\Delta}^{\perp}}^{m-1} X(j), \Delta_{X_{\Delta}^{\perp}}^{m} X(j)\right) .
\end{aligned}
$$

By property 1 we can conclude that $\forall i, n \in \mathbb{Z}$, we have $\Delta_{X_{\Delta}}^{n} X(i)=\tau_{X}^{i} X_{\Delta}^{\perp}(n)$.

Corollary 1. Let $X \in \mathcal{C}_{N}^{\pi}$ reproducible over $\Delta$ (respectively $\tau$ ) in $\delta$ steps. Then there exists a unique periodic dual sequence $X_{\Delta}^{\perp}$ (respectively $X_{\mathcal{\tau}}^{\perp}$ ); this sequence is of cyclicity $\delta$ and is reproducible over $\tau$ (respectively $\Delta$ ) in $\pi$ steps.

Property 3. Let $X \in \mathcal{C}_{N}$. We have, $\forall n \in \mathbb{N}, i \in \mathbb{Z}$ :

$$
\begin{aligned}
& \Delta^{n} X(i)=\sum_{j=0}^{n}(-1)^{n-j}\left(\begin{array}{l}
n \\
j
\end{array}\right) X(i+j) \\
& \tau^{n} X(i)=\sum_{j=0}^{n}\left(\begin{array}{l}
n \\
j
\end{array}\right) X(i+j)
\end{aligned}
$$


Proof. Since $\left(\mathbb{Z}\left[\sigma^{ \pm 1}\right], \circ\right)$ is commutative, we can use the binomial theorem :

$$
\begin{aligned}
& \Delta^{n}=(\sigma-i d)^{n}=\sum_{j=0}^{n}(-1)^{n+j}\left(\begin{array}{c}
n \\
j
\end{array}\right) \sigma^{j} \\
& \boldsymbol{\tau}^{n}=(\sigma+i d)^{n}=\sum_{j=0}^{n}\left(\begin{array}{c}
n \\
j
\end{array}\right) \sigma^{j}
\end{aligned}
$$

and the formulae follow by the left distributivity of application.

\section{Lemma 1.}

$$
\forall k \geq 0, \forall i, n \text { such that } i \geq n: \sum_{j=n}^{i}(-1)^{j}\left(\begin{array}{c}
i \\
j
\end{array}\right)\left(\begin{array}{c}
j-n \\
k
\end{array}\right)=(-1)^{n+k}\left(\begin{array}{c}
i-k-1 \\
n-1
\end{array}\right) .
$$

Proof.

$$
\begin{aligned}
& A_{i, k}:=\sum_{j=n}^{i}(-1)^{j}\left(\begin{array}{c}
i \\
j
\end{array}\right)\left(\begin{array}{c}
j-n \\
k
\end{array}\right)=\sum_{j=n}^{i}(-1)^{j}\left[\left(\begin{array}{c}
j \\
i-1
\end{array}\right)+\left(\begin{array}{l}
j-1 \\
i-1
\end{array}\right)\right]\left(\begin{array}{c}
k \\
j-n
\end{array}\right) \\
& =\sum_{j=n}^{i}(-1)^{j}\left(\begin{array}{c}
j \\
i-1
\end{array}\right)\left(\begin{array}{c}
k \\
j-n
\end{array}\right)+\sum_{j=n-1}^{i-1}(-1)^{j+1}\left(\begin{array}{c}
j \\
i-1
\end{array}\right)\left(\begin{array}{c}
k \\
j+1-n
\end{array}\right)
\end{aligned}
$$

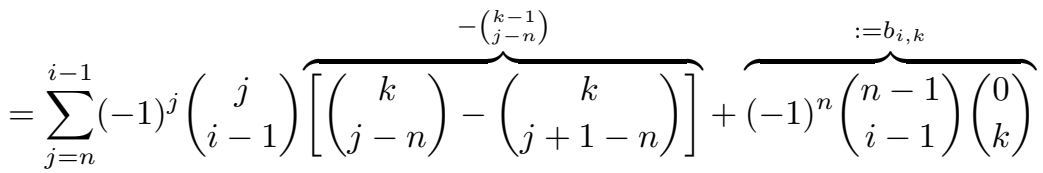

$$
\begin{aligned}
& =-A_{i-1, k-1}+b_{i, k}=\sum_{q=0}^{k}(-1)^{q} b_{i-q, k-q}=(-1)^{k} b_{i-k, 0} \\
& =(-1)^{k+n}\left(\begin{array}{c}
n-1 \\
i-k-1
\end{array}\right) \text { by definition. }
\end{aligned}
$$

Note that $A_{i-q, q}=0$ when $q>k$ and that $\forall i: b_{i, k}=0$ for $k \neq 0$.

Property 4. Let $X \in \mathcal{C}_{N}$ and $A=\left(a_{1}, \ldots, a_{n}\right) \in \mathbb{Z}_{N}^{n}$. The values of antecedents are given by the following formulae :

$$
\begin{aligned}
& \Delta_{A}^{-n} X(i)=\sum_{j=0}^{n-1}\left(\begin{array}{l}
i \\
j
\end{array}\right) X_{\Delta}^{\perp}(n-j)+\sum_{k=0}^{i-n}\left(\begin{array}{c}
i-k-1 \\
n-1
\end{array}\right) X(k) \\
& \tau_{A}^{-n} X(i)=\sum_{j=0}^{i}(-1)^{i+j}\left(\begin{array}{c}
i \\
j
\end{array}\right) X_{\bar{\tau}}^{\perp}(n-j)+\sum_{k=0}^{i-n}(-1)^{k+i+n}\left(\begin{array}{c}
i+k-1 \\
n-1
\end{array}\right) X(k)
\end{aligned}
$$


Proof. By the duality of $\tau$ and $\Delta$, we have :

$$
\begin{aligned}
\Delta_{A}^{-n} X(i) & =\tau^{i} X_{\Delta}^{\perp}(0)=\sum_{j=0}^{i}\left(\begin{array}{c}
i \\
j
\end{array}\right) X_{\Delta}^{\perp}(j)=\sum_{j=0}^{n-1}\left(\begin{array}{c}
i \\
j
\end{array}\right) X_{\Delta}^{\perp}(n-j)+\sum_{j=n}^{i}\left(\begin{array}{c}
i \\
j
\end{array}\right) \Delta^{j-n} X(0) \\
& =\sum_{j=0}^{n-1}\left(\begin{array}{l}
i \\
j
\end{array}\right) X_{\Delta}^{\perp}(n-j)+\sum_{j=n}^{i}\left(\begin{array}{l}
i \\
j
\end{array}\right) \sum_{k=0}^{j-n}(-1)^{j-n+k}\left(\begin{array}{c}
j-n \\
k
\end{array}\right) X(k) \\
& =\sum_{j=0}^{n-1}\left(\begin{array}{l}
i \\
j
\end{array}\right) X_{\Delta}^{\perp}(n-j)+\sum_{k=0}^{i-n}(-1)^{-n+k}\left[\sum_{j=n}^{i}\left(\begin{array}{c}
i \\
j
\end{array}\right)(-1)^{j}\left(\begin{array}{c}
j-n \\
k
\end{array}\right)\right] X(k)
\end{aligned}
$$

Similarly, we have :

$$
\begin{aligned}
\boldsymbol{\tau}_{A}^{-n} X(i) & =\Delta^{i} X_{\boldsymbol{\tau}}^{\perp}(0)=\sum_{j=0}^{i}(-1)^{i+j}\left(\begin{array}{l}
i \\
j
\end{array}\right) X_{\boldsymbol{\tau}}^{\perp}(j) \\
& =\sum_{j=0}^{n-1}(-1)^{i+j}\left(\begin{array}{l}
i \\
j
\end{array}\right) X_{\boldsymbol{\tau}}^{\perp}(n-j)+\sum_{j=n}^{i}(-1)^{i+j}\left(\begin{array}{c}
i \\
j
\end{array}\right) \boldsymbol{\tau}^{j-n} X(0) \\
& =\sum_{j=0}^{n-1}(-1)^{i+j}\left(\begin{array}{l}
i \\
j
\end{array}\right) X_{\boldsymbol{\tau}}^{\perp}(n-j)+\sum_{j=n}^{i}(-1)^{i+j}\left(\begin{array}{c}
i \\
j
\end{array}\right) \sum_{k=0}^{j-n}\left(\begin{array}{c}
j-n \\
k
\end{array}\right) X(k) \\
& =\sum_{j=0}^{n-1}(-1)^{i+j}\left(\begin{array}{c}
i \\
j
\end{array}\right) X_{\boldsymbol{\tau}}^{\perp}(n-j)+\sum_{k=0}^{i-n}(-1)^{i}\left[\sum_{j=n}^{i}\left(\begin{array}{c}
i \\
j
\end{array}\right)(-1)^{j}\left(\begin{array}{c}
j-n \\
k
\end{array}\right)\right] X(k)
\end{aligned}
$$

By applying lemma 1 to the expression in square brackets in both equations, we obtain the aforementionned formulae.

Unlike through $F, \mathcal{C}_{N}^{\pi}$ is not stable through $F_{a}^{-1}$, as the period may grow, in a fashion we will now characterise.

Theorem 6. Let $X \in \mathcal{C}_{N}$ of period $\tau$. Then $\Delta_{a}^{-1} X$ is of period $o_{N}(\bar{X}) \tau$, where $o_{N}(x)$ denotes the (additive) order of $x$ in $\mathbb{Z}_{N}$, and $\bar{X}=\Delta_{a}^{-1} X(\tau)-\Delta_{a}^{-1} X(0)$.

Proof. Since $X$ is $\tau$-periodic, $\forall i, k \in \mathbb{Z}, X(i)=X(i+k \tau)$, therefore $\Delta_{a}^{-1} X(i+$ $k \tau)=\Delta_{a}^{-1} X(i) \Longleftrightarrow \Delta_{a}^{-1} X(i+k \tau+1)=\Delta_{a}^{-1} X(i+1)$ and by induction $\Delta_{a}^{-1} X$ is $k \tau$-cyclic. We also know that $\tau$ divides the period of $\Delta_{a}^{-1} X$ so in order to find it we need to find the minimal integer $k>0$ such that $\Delta_{a}^{-1} X(k \tau)=\Delta_{a}^{-1} X(0)$.

From the formula above, we can see that $\Delta_{a}^{-1} X(i+\tau)-\Delta_{a}^{-1} X(i)=\Delta_{a}^{-1} X(i+$ $n \tau)-\Delta_{a}^{-1} X(i+(n-1) \tau)$, therefore $\Delta_{a}^{-1} X(k \tau)-\Delta_{a}^{-1} X(0)=k\left(\Delta_{a}^{-1} X(k \tau)-\right.$ $\left.\Delta_{a}^{-1} X(0)\right)$. By definition, the smallest $k>0$ such that this product is 0 is the order of $\bar{X}$ (as defined above), hence $\Delta_{a}^{-1} X$ is $o_{N}(\bar{X}) \tau$-periodic.

Theorem 7. Let $X \in \mathcal{C}_{N}$ of period $\tau$. Then $\boldsymbol{\tau}_{a}^{-1} X$ is of period: 


$$
\begin{aligned}
& X \mid 1355101355101 \ldots \\
& \Delta_{2}^{-1} X \mid 2305455032122 \ldots
\end{aligned}
$$

Fig. 3: Period growth on a $\mathbb{Z}_{6}$-valued sequence.

- $o_{N}(\bar{X}) \tau$, where $\bar{X}=\boldsymbol{\tau}_{a}^{-1} X(\tau)-\boldsymbol{\tau}_{a}^{-1} X(0)$, if $\tau$ is even.

- $\tau$ if $\bar{X}=0$ or $2 \tau$ otherwise, if $\tau$ is odd.

Proof. Through the same argument as above, we need to find the smallest integer $k>0$ such that $\boldsymbol{\tau}_{a}^{-1} X(k \tau)=\boldsymbol{\tau}_{a}^{-1} X(0)$. From there two cases arise:

- $\tau$ is even : from the formula, we get $\Delta_{a}^{-1} X(i+\tau)-\Delta_{a}^{-1} X(i)=\Delta_{a}^{-1} X(i+$ $n \tau)-\Delta_{a}^{-1} X(i+(n-1) \tau)$ and the rest of the proof follows as above;

- $\tau$ is odd : we get $\Delta_{a}^{-1} X(i+\tau)-\Delta_{a}^{-1} X(i)=-\left(\Delta_{a}^{-1} X(i+2 \tau)-\Delta_{a}^{-1} X(i+\tau)\right)$, therefore $\Delta_{a}^{-1} X(i+2 \tau)=\Delta_{a}^{-1} X(i)$ and $k$ is 2 , unless $\bar{X}=0$, in which case $k$ is 1 .

This last result has interesting corollaries that allow us to partially characterise reproducible and reducible sequences over $\tau$.

Corollary 2. If a sequence $X \in \mathcal{C}_{N}$ is reducible over $\boldsymbol{\tau}$, then it is either constant or its period is of the form $2 \prod_{k} q_{k}$, where $q_{k}$ divides $N$.

Proof. Any reducible sequence is an antecedent of the zero sequence, which is of period 1 , and the order of an element of $\mathbb{Z}_{N}$ always divides $N$.

Corollary 3. If a sequence $X \in \mathcal{C}_{N}^{\pi}$ is reducible over $\tau$ and $\pi$ is odd, then it is constant and $X(0) \in \frac{N}{2^{k}} \mathbb{Z}_{N}$ with $2^{k}$ the largest power of 2 dividing $N$.

Proof. $X$ is constant according to the previous corollary. The value of constant sequences doubles with each iteration of $\tau$, and $\frac{N}{2^{k}} \mathbb{Z}_{N}$ are the solutions to the equation $\exists l: 2^{l} \times x=0 \bmod N$.

Corollary 4. Let $X \in \mathcal{C}_{N}^{\pi}$. If $N$ and $\pi$ are odd, then $X$ is reproducible over $\tau$.

Proof. From the previous corollary, if both $N$ and $\pi$ are odd, the only reducible sequence is the zero sequence. Therefore, $\mathcal{C}_{N}^{\pi}=\operatorname{Rep} \boldsymbol{\tau}\left(\mathcal{C}_{N}^{\pi}\right) \oplus\left\{0^{\mathbb{Z}}\right\}=\operatorname{Rep} \tau\left(\mathcal{C}_{N}^{\pi}\right)$.

\section{Musical example}

The search of the preimages leads to a natural question from a compositional point of view: can we find elements of our initial periodic sequence by going in its past with the two automata or do we forget totally our initial configuration?

The chosen example is linked to that question, being a fugue, whose sheet: 5 and audi ${ }^{6}$ are available online. In fact in a fugue, a leading element is present, the so called subject that may be accompanied by one or more countersubjects.

5 http://repmus.ircam.fr/_media/moreno/score_lanthier_duality.pdf

6 http://repmus.ircam.fr/_media/moreno/audio_lanthier_duality.mp3 
After the exposition where the subject and countersubjects move from one part to another, a special moment arises: the development. This moment is interesting and one of the main possible challenges is to bring something new but still inherited from the subject and countersubjects.

The alphabet $\mathcal{A}=\mathbb{Z}_{16}$ was chosen with the scale represented in figure 4

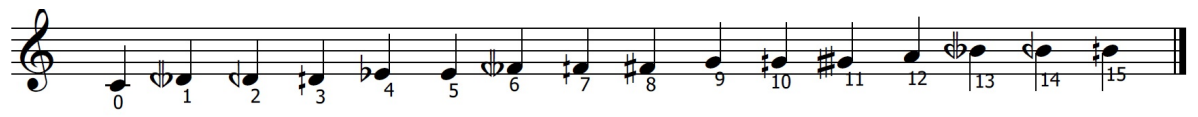

Fig. 4: The octave divided in 16 equal parts which is used for the composition.

A subject $\alpha$ was chosen to lead this experience and the original thing was to take two countersubjects being the images of this subject by the two automata.

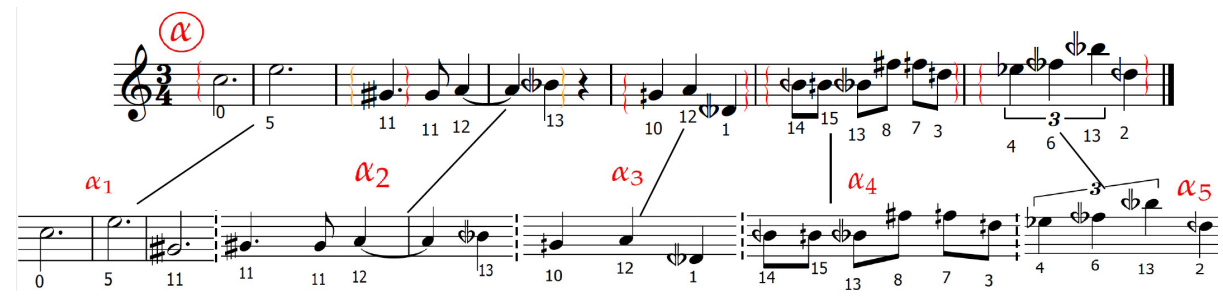

Fig. 5: The thema "Alpha"

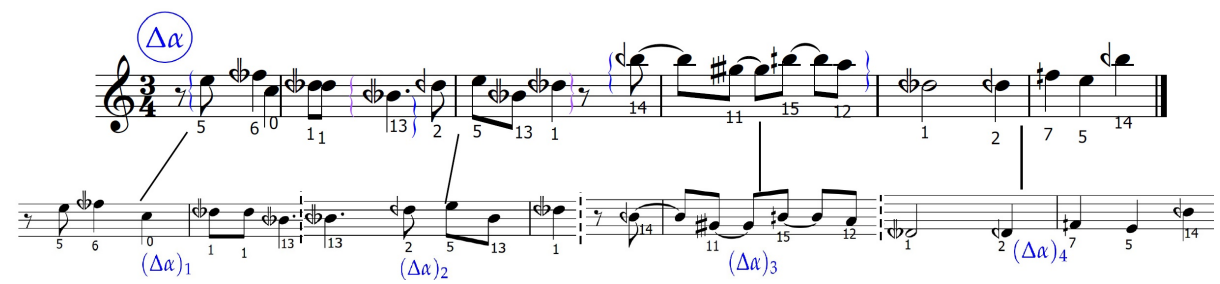

Fig. 6: First countersubject

To find elements of $\alpha, \Delta \alpha$ and $\tau \alpha$ in the preimages, the search of permutations (noted p), transpositions $(\alpha+a)$, subsequences $(\eta)$ and "similar" elements were used, for instance $10,13,1$ is considered close to $10,12,1$ which we indicate by $10,13,1 \simeq 10,12,1$. another symbol was used to describe the "head" of a thema, noted $\wedge$. 


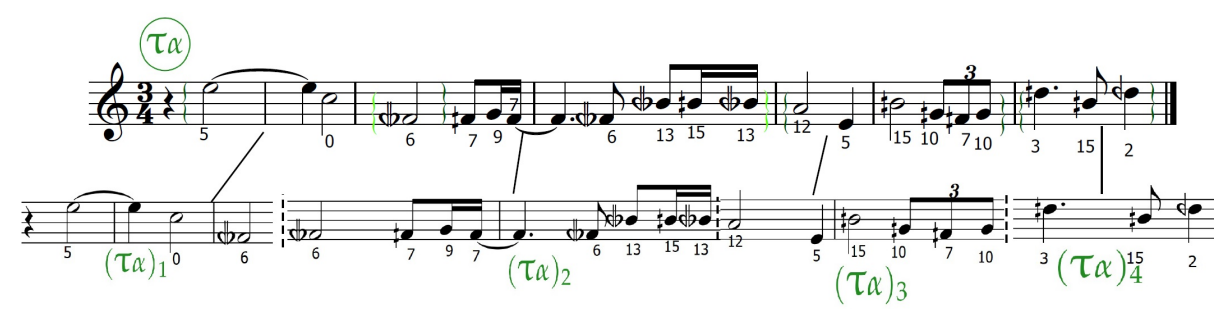

Fig. 7: Second countersubject

We can clearly see that the density of elements of $\alpha, \Delta \alpha$ and $\tau \alpha$ in the development is high in respect to the transformations we spoke about. This clearly leads to think that there is a weaker notion than reproducibility in the past to investigate and that the initial value is not forgotten in a sense. The preimages were chosen for the period to remain constant or to double in order to not have too long thema, it constrained the number of possibilities.

\section{Perspectives}

An important open question would be to demonstrate if by choosing some specific initial values preimages can be obtained containing more elements of the initial configuration than some other paths. From a probabilistic point of view, it would be interesting to see if by choosing randomly the initial values of the preimages, we forget the initial configuration. The main difficulty lies in the evolutionary character of the period of the Markov process that the preimages describe. But in the case of a non-exploding period evolution we can conjecture that the initial condition is eventually forgotten, as the uniform measure on the alphabet used to choose initial values for preimages is preserved by the two automata combined with the duality result. Do other pairs of cellular automata share a similar duality? Is it possible to generalize Vieru's theory via wider classes of automata?

\section{References}

1. Ancellotti, N.: On Some Algebraic Aspects of Anatol Vieru Periodic Sequences. Master, Università degli studi di Padova (2015)

2. Andreatta, M., Vuza, D.T.: On some properties of periodic sequences in Anatol Vieru's modal theory. Tatra Mt. Math. Publ 23(1), 1-15 (2001)

3. Andreatta, M., Vuza, D.T., Agon, C.: On some theoretical and computational aspects of Anatol Vieru's periodic sequences. Soft Computing 8(9), 588-596 (2004)

4. Vieru, A.: Modalism-A" Third World". Perspectives of New Music pp. 62-71 (1985)

5. Vieru, A.: The Book of Modes (I, II): From Modes to a Model of the Intervallic Musical Thought: from Modes to Musical Time. Editura Muzicală (1993)

6. Vuza, D.: Aspects mathématiques dans la théorie modale d'Anatol Vieru. Editura Academiei Republicii Socialiste România (1982) 

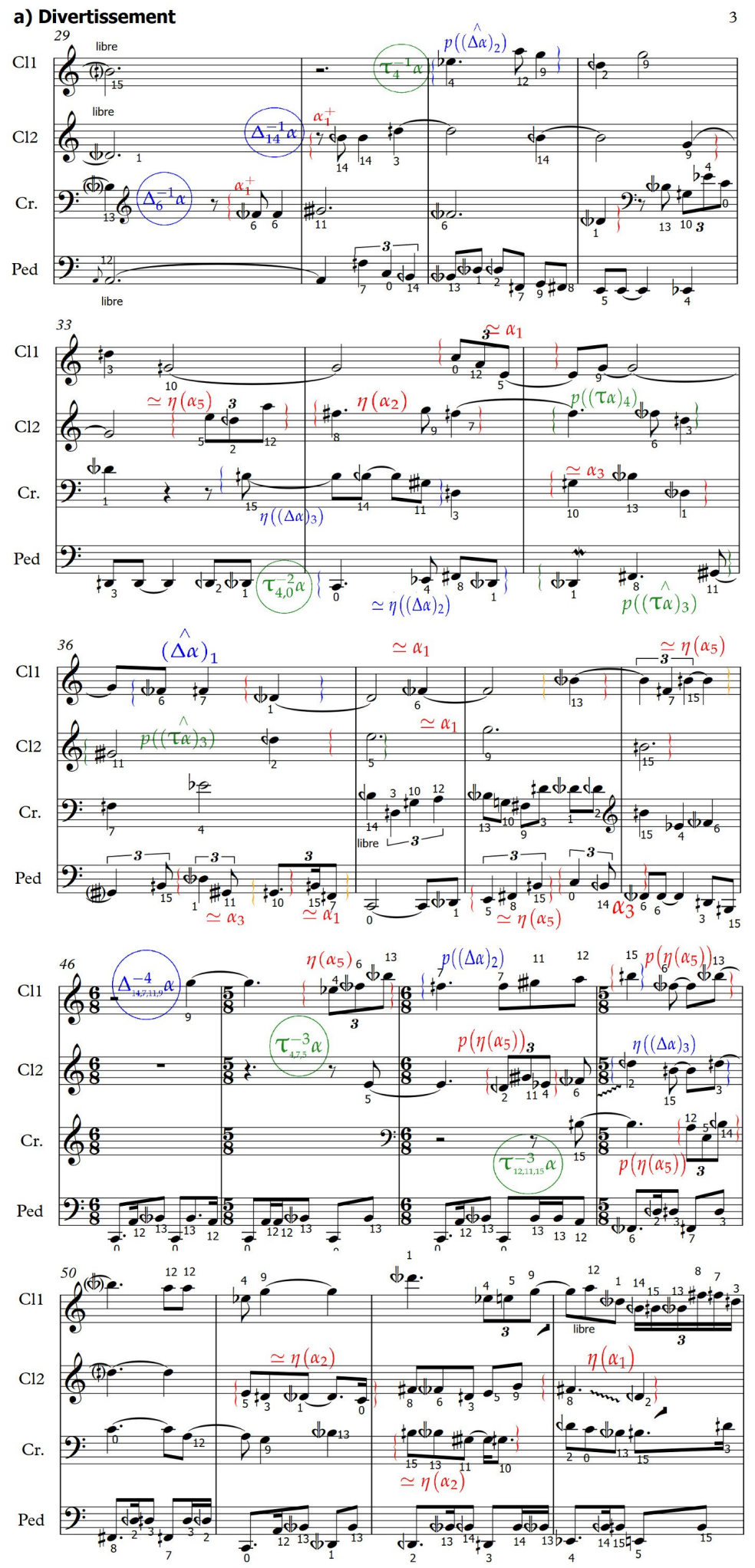

Fig. 8: 2 Extracts $[29,39]$ and $[46,53]$ bars from the development with preimages 